\title{
Carrots, Sticks and Fog During Insurgencies
}

\author{
Michael P. Atkinson \\ Moshe Kress \\ Roberto Szechtman \\ mpatkins@nps.edu \\ mkress@nps.edu \\ rszechtm@nps.edu \\ Operations Research Department \\ Naval Postgraduate School \\ Monterey CA 93943
}

March 21, 2012

\begin{abstract}
We formulate a rational choice model of popular behavior during an insurgency. An individual in the population either supports the insurgents or the government depending upon his attitude and the actions taken by each side. We focus on the effect of insurgency actions: benefits, impositions, and coercion. While benefits and impositions are applied uniformly throughout, the insurgents intend to only coerce those actively providing information to the government. However, due to the "fog of war", which may lead to limited situational awareness, the insurgents may mistakenly coerce their own supporters and potentially drive them to aid the government. We examine how popular behavior varies under different situational awareness scenarios. When the insurgents have little situational awareness, they should take few coercive actions. This implies that the government will be able to foster intelligence sources within the population. If the insurgents have perfect situational awareness, tipping points may occur that result in a significant reduction in active support for the government. In this case the government should take actions to decrease the coercing effectiveness of the insurgents and increase incentives to the population so they continue to provide information.
\end{abstract}




\section{Introduction}

Several recent military conflicts (Somalia, Iraq, Afghanistan, Southern Lebanon) differ from traditional force-on-force engagements, taking the form of an asymmetrical counterinsurgency situation where the civilian population plays a major role. Consequently, legacy warfare and combat models (e.g., Lanchester models (Washburn and Kress, 2009)) must be updated to reflect this significant shift in conflict paradigm. In particular, the increasingly important part of the population must be explicitly modeled. Almost all current research efforts in counterinsurgency modeling concentrate on simulations (e.g., Farley (2007)) - primarily agent-based models (e.g., Epstein (2002), Bhavnani et al. (2008), Cioffi-Revilla and Rouleau (2010)). Counterinsurgency simulations typically rely on a large amount of data, which is often incomplete, noisy, or all-together unavailable. In contrast, we propose in this paper a macroscopic and analytic model that relies on general and robust qualitative assumptions, and on a relatively small number of key parameters that can be estimated from available data or expert opinions. The objective of the model is to provide transparent cause-and-effect insights about general relations in counterinsurgency situations, without imposing unreasonable demands on data. The model focuses on the effect of insurgency actions, in particular coercion, on the population's behavior. Using a set of basic assumptions, rooted in the social sciences literature, regarding population behavioral patterns, we construct a utility-based model that evaluates the effects of insurgency actions and produces robust insights.

We consider a counterinsurgency situation motivated by the one currently (2011) in progress on the Pakistan/Afghanistan border, in which insurgents are in control in some rural region where the government has limited or no control. The insurgents "call the shots" and the civilian population in the region reacts to these actions based on their perception regarding the capabilities and intentions of the government to improve or worsen their welfare. The situation comprises three major players: insurgents, government and civilian population. The insurgents are motivated by ideological, social, political, and economic goals, while the objective of the government is to eliminate the insurgency. The population's behavior towards the insurgents and government, which may depend on ethnic, religious, social and economic characteristics of the population, is central; both the insurgents and the government fight for the "hearts and minds" of the population. For the insurgency, the population is a source of recruitment, economic and material support, and information. The insurgents also may force ideological, political and cultural impositions on the population. For the government, the population is a source of intelligence about the insurgents, which may be utilized for better targeting the insurgents. Thus, the insurgency thrives when the population is on its side - providing support to the insurgents and favorably responding to ideological impositions - and tends to decline when the population supports the government and provides it with intelligence.

In this paper we study the effect of insurgency actions on the behavior of the population towards the insurgents and the government. The insurgents target some of their actions on certain groups in the population. and the success of this targeting depends on the situational awareness of the insurgents. Situational awareness (SA) is a measure of the accuracy in which the insurgents target their actions. While we model the situation for any level of SA, we study in detail the two extreme cases: (a) no SA, where no targeting is possible, and (b) perfect 
SA where the insurgents can target their actions with perfect accuracy. One objective is to study the type of actions "rational" insurgents may take, and the policy implications of such actions for the government. Another objective is to identify conditions where small changes in the actions of the insurgents can affect behavior that rapidly propagates through the entire population causing a dramatic shift in popular behavior (Granovetter, 1978). These "tipping points" lead to abrupt and extreme changes in population behavior. Tipping points can occur in many other social situations (e.g., crime rates in a city (Kleiman and Kilmer, 2009) and the demographics of a suburb (Card et al., 2008)).

In a simple and compelling model, Kress and Szechtman (2009) show that it is nearly impossible to eradicate insurgents in the absence of "soft power." In that paper only a homogeneous insurgency is considered, and the population behavior, which is only implicitly represented in the model, is consistent throughout. Berman et al. (2008) further highlight the importance of soft actions that lead to less violence. Another empirical work shows that revenge by people for civilian casualties results in increased insurgents violence over the long-run (Condra et al., 2010). The approach adopted in our paper is rooted on rational choice theory, and builds upon the approach presented in Akerlof and Yellen (1994), which studies gang behavior in urban areas.

We note the difference between the attitude of an individual and his behavior. For example, a person who fundamentally opposes to the insurgents (attitude) may express latent or active opposition to the insurgents (behavior) based on pragmatic considerations. The terms attitude and behavior used here are related, respectively, to the terms "private preference" and "public preference" used in Kuran (1989). While it may be hard to shape the attitude of people - their fundamental beliefs and values that have been shaped over centuries of cultural evolution - it may be possible to affect their manifested behavior, which is influenced by interests and utilities. Also note that the objective of the insurgents is to deter potential supporters of the regime. However, unlike typical deterring settings (Zagare and Kilgour, 2000) the deterrence is one-way: deterring civilians from providing information to the regime.

Several operational insights follow from analyzing the proposed model. These insights address the existence of equilibria in population behavior, the conditions and the nature of tipping points, and the properties of an optimal mix of actions carried out by insurgents, which are subject to limited resources.

In Section 2 we describe the operational setting and the basic assumptions used to formulate the model. Section 3 presents the model and discusses its specific assumptions. In particular, we discuss in this section convergence, equilibrium points and the insurgents' feasible actions. In Section 4 we discuss the issue of situational awareness and examine in detail the two extreme cases: no SA and perfect SA. Section 5 provides some conclusions and policy implications.

\section{Operational Setting and Basic Assumptions}

We assume that the population is rational in the sense that an individual favors whomever offers the highest utility. This utility depends on the individual's attitude, which is derived from his specific background, ideology, objectives, and preferences. For example, a devout 
Muslim would prefer a strict religious school curriculum for his children over a more general and secular one offered by the government, while a secular person may prefer otherwise. Thus, the former may tolerate a higher level of religious impositions by the insurgents than the latter, and this fact will be manifested in their respective behaviors towards the insurgents.

An individual in the population is faced with a set of actions presented by the insurgents, and a reference point representing his perception or expectation regarding the utility that he could derive from the government, if it were in power. That individual chooses the best of these two alternatives. Hence, at any given time the population is divided into two major groups, possibly depending on ethnicity, religious beliefs, and social and economic status: Supporters of the insurgents and non-supporters. The latter are called henceforth contrarians, who feel they would be better off with the government in place.

The insurgents apply two types of actions: non-violent and violent acts of coercive nature. Non-violent actions befall the population at large, and may strengthen the insurgency by promoting its status and increasing its power, or may weaken the insurgency if they are too costly or unpopular with the population. Non-violent actions consist of benefits and impositions. Benefits, which may include providing security, health care, and education, represent investments by the insurgents to gain popular support. Ceteris paribus, benefits cannot reduce the utility of an individual; more benefits are better than less. On the other hand, impositions are designed to generate direct benefits for the insurgents by supporting their political and ideological agenda (e.g., enforcing certain school curricula, dress code for women) and enhancing their economic and physical wherewithal (e.g., taxation, recruitment). While in some cases some impositions may enhance the utility of an individual (e.g., a devout Muslim may prefer stricter Islamic rules than lenient ones) we assume, for simplicity, that the utility of an individual is non-increasing in the impositions. Moreover, we assume that the net effect of the insurgents' non-violent actions is represented by a scalar parameter, called net-benefits; a negative value indicates excess impositions (e.g., taxes) while a positive value indicates more benefits.

Coercive actions by the insurgents are intended to prevent contrarians from cooperating (e.g., snitching on insurgents' activities) with the government. Coercion comprises violent actions (or threats thereof) such as kidnapping, torture and killing; higher coercion levels increase fear and thus may make people reluctant to provide information to the government. We represent the coercion by a non-negative scalar parameter. Unlike non-violent actions that are uniformly applied on the entire population, the insurgents only intend to coerce contrarians who aid the government. However, the insurgents may not have perfect information (SA) about the identity, supporter or contrarian, of each individual in the population and therefore may inadvertently coerce supporters (e.g., kidnap the wrong person).

The effect of coercion may vary; some contrarians may be coerced and become latent contrarians, while others will defy the insurgents and will remain active contrarians. In the case of perfect SA, the coercive actions by the insurgents are fully and effectively targeted only at the active contrarians, aiming at making them latent. However, if the insurgents have imperfect SA about the population, only a fraction of the coercive actions falls on the active contrarians; the rest of the coercive effort falls mistakenly on the supporters and latent contrarians.

Since supporters may be the recipient of unintended coercion, the fraction of supporters 
is determined by both the net-benefits and the level of coercion. Individuals who receive less utility from the actions carried out by the insurgents (net-benefits and coercion) than their perceived utility from the government become contrarians. Otherwise, they are supporters of the insurgents. Contrarians make another utility comparison to decide whether they should be active or latent.

Therefore, the decision to be an active or latent contrarian is a two step process: first an individual chooses to become a contrarian because he expects to be better off with the government, then he evaluates whether to bear the coercion and actively help the government or become latent. The utility of an individual in the population depends on his private preferences (i.e., attitude) and the set of actions carried out by the insurgents. The private preferences of an individual, based on tradition, narratives and cultural values, determine his utility function. This (individual) function, which varies in the population according to some probability distribution, remains unchanged throughout the time horizon modeled. The actions of the insurgents are the arguments of this utility function and, together with the perceived utility from the government, they determine the public preferences or behavior of that individual. The behavior of an individual is manifested in being a supporter, a latent contrarian, or an active contrarian. The primary objective of the insurgents is to have no active contrarians, and the secondary objective is to maximize the number of supporters in the population.

The net-benefits and coercion are varying parameters in our model chosen by the insurgents, whereas the reference utility associated with the government remains constant throughout. The set of insurgents' actions - non-violent and coercive - is bounded by the insurgents' strength, available resources and perhaps self-imposed limitations; the insurgents must trade off the resources and efforts they put into non-violent actions and coercion.

\section{The Model}

The set of actions generating net-benefits are expressed by the parameter $x$, and the coercive actions, which are targeted on active contrarians, are represented by the non-negative parameter $y$. The insurgents choose the values of $x$ and $y$. Targeting may not be effective due to imperfect situational awareness. Faced with insurgents' actions $(x, y)$, an individual computes his utility from these actions, compares it with his perceived utility from the government, and decides whether to support the insurgents or oppose them. If he chooses the latter, then he has to decide whether to be latent or active.

Let $S(x, y), C(x, y), L(x, y)$ and $A(x, y)$ denote the fraction of supporters, contrarians, latent contrarians and active contrarians, respectively when the insurgents execute actions $(x, y), C(x, y)=L(x, y)+A(x, y), S(x, y)+C(x, y)=1$. To simplify the notation we often drop the $(x, y)$ dependency from $S, C, L$, and $A$ whenever the exposition remains apparent. We focus our analysis on $S$ and $A$ because $C$ and $L$ can be computed from those two quantities. Let $u(x, y ; \omega)$ denote the utility of an individual (indexed as $\omega$ ) faced with $x$ net-benefits and $y$ coercion, and let $\alpha(\omega)$ denote the utility surmised by that individual from the government. We view $u(x, y)$ and $\alpha$ as random variables defined on the population space. The net-benefits are uniformly spread among the entire population, whereas the coercion is targeted on the active contrarians. We assume that, for every $\omega, u(x, y ; \omega)$ is differentiable, 
non-decreasing in $x$, non-increasing in $y$, and bounded for $(x, y)$ finite.

While the insurgents aim to only coerce the active contrarians, some supporters and latent contrarians will mistakenly get a share of the coercion because of the imperfect situational awareness of the insurgents. Let $y_{s \ell}$ denote the share of coercion unintentionally applied to supporters and latent contrarians, and let $y_{a}$ denote the coercion share carried out against the active contrarians, $y_{s \ell}+y_{a}=y$. Both coercion shares, $y_{s \ell}$ and $y_{a}$, depend on the fraction of active contrarians $A$. It is reasonable to assume that as this fraction increases, the signature of the active contrarians becomes larger. Therefore, $y_{a}(A)$ is monotone non-decreasing and consequently $y_{s \ell}(A)$ is non-increasing. The per-capita net-benefits is $x$ (because the population size is normalized to 1 ) and the per-capita coercion that (unintentionally) falls on a supporter or a latent contrarian is $y_{s \ell}(A) /(1-A)$.

Observing the $(x, y)$ values, the individual denoted by $\omega$ is a supporter if and only if

$$
u\left(x, \frac{y_{s \ell}(A)}{1-A} ; \omega\right) \geq \alpha(\omega)
$$

that is, if the utility received from the insurgents is higher than the perceived utility that individual expects had the government been in control.

The successful targeting of coercion depends on the situational awareness of the insurgents regarding the identity of the active contrarians. Under any realistic assumption about the situational awareness (see assumption A.3 below), an individual receives higher utility as a latent contrarian than as an active one. However, based on his private preferences (attitude), a contrarian may opt to become active notwithstanding the lower utility he may incur. Let $\beta(\omega) \geq 0$ denote the amount of disutility an individual $(\omega)$ is willing to bear to help the government. This threshold may account for the individual's integrity in standing up for what he believes in (see e.g., Kuran (1989)), or the belief that by helping the government in the present, and bearing the coercion of the insurgents, the individual or his family will have a better life in the future if the insurgents are defeated. Similarly to $u(x, y)$ and $\alpha, \beta$ is also a random variable defined on the space of individuals in the population. An individual is an active contrarian if

$$
u\left(x, \frac{y_{s \ell}(A)}{1-A} ; \omega\right)<\alpha(\omega) \text { and } u\left(x, \frac{y_{s \ell}(A)}{1-A} ; \omega\right)-u\left(x, \frac{y_{a}(A)}{A} ; \omega\right)<\beta(\omega) .
$$

We now present the mathematical assumptions used for the analysis of our model; the operational motivation for these assumptions appears thereafter.

A.1. The per-capita coercion applied on the active contrarians, $y_{a}(A) / A$, is non-increasing in $A$.

A.2. The per-capita coercion applied on the supporters and latent contrarians, $y_{s \ell}(A) /(1-$ $A)$, is non-decreasing in $A$.

A.3. The per-capita coercion falling on the active contrarians, $y_{a}(A) / A$, is at least $y$.

A.4. $P\left[u\left(x, y_{s \ell}(A) /(1-A)\right)=\alpha\right]=0$ holds for every feasible value of $x, y$ and $A \in(0,1]$.

A.5. $P\left[u\left(x, y_{s \ell}(A) /(1-A)\right)-u\left(x, y_{a}(A) / A\right)=\beta\right]=0$ holds for every feasible value of $x, y$ and $A \in(0,1]$. 
A.6. $P\left[u\left(x, y_{s \ell}(A) /(1-A)\right)<\alpha ; u\left(x, y_{s \ell}(A) /(1-A)\right)-u\left(x, y_{a}(A) / A\right)<\beta\right]$ is continuous from the right at $A=0$.

A.7. For each individual $\omega$, we have $\partial u\left(x, y_{1} ; \omega\right) / \partial x \geq \partial u\left(x, y_{2} ; \omega\right) / \partial x$ for $y_{1} \leq y_{2}$.

Assumptions A.1 and A.2 essentially assert that the effect of the population size of a certain sector $(S, L$ or $A$ ) overrides the effect of enhanced situational awareness. In other words, the additional coercion targeted correctly on $A$ due to a larger signature has a lesser effect than the increased number of individuals who share this coercion. Thus, the larger the active opposition, the lower the average cost of being an active, and the higher the average cost of not being one.

Assumption A.3 specifies that the worst-case for the insurgents, in terms of coercion, is to "shoot in the dark." If the insurgents coerce people at random, they will still pick a fraction $A$ of active contrarians by chance. Even though it is possible that the active contrarians (or the government) may attempt to use deception to alter the insurgent's perception about the behavior of individuals in the population, we assume that in practice such an effect would be marginal at best. Thus, it is not realistic for the insurgents to coerce in a less effective manner than picking people at random. This condition also implies that $\frac{y_{s \ell}(A)}{y} \leq 1-A$ and thus

$$
\frac{y_{s \ell}(A)}{1-A} \leq y \leq \frac{y_{a}(A)}{A}
$$

Therefore active contrarians receive higher per-capita coercion than latent contrarians or supporters and it is more costly to be an active contrarian than a latent one.

Assumptions A.4-A.6 are technical assumptions that ensure continuity of functions that we analyze in the following sections. We use Theorem 7.43 in Shapiro et al. (2009) to prove continuity and conditions A.4 and A.5 are necessary to utilize that theorem. If these conditions do not hold, it is possible that a non-trivial fraction of the population reaches the indifference point (either between contrarian and supporter or between active and latent) for the same action combination $(x, y)$. An example of such discontinuity would be a clan of individuals who all have exactly the same utility function. In section 4.2 .2 we present another type of discontinuity where cascading changes in the population lead to a large shift in popular behavior.

Assumption A.7 states that people's sensitivities to net-benefits decrease with the level of coercion. This means that marginal changes in net-benefits matter more to individuals who receive less coercion. Conversely, the more coercion an individual receives, the less he cares about net-benefits. While this condition may not hold true for all values of $(x, y)$ (e.g., large values of $x$ ), this is a reasonable assumption for an insurgency situation where the insurgents' ability to execute actions is limited (see section 3.2).

\subsection{Convergence and Equilibrium}

In this section, we assume the insurgents choose a value of $x$ and $y$, and we then examine the population dynamics for these fixed values of $x$ and $y$. Faced with insurgents' actions $(x, y)$, the situation evolves in stages $n=1,2, \ldots$. At each stage, individuals maximize their single stage utility given the knowledge regarding the per-capita coercion received by each group (supporter, latent contrarian, or active contrarian) in the previous stage. This suggests that 
the iterative process driven by the individuals' best responses results in the convergence of the fraction of supporters, latent contrarians, and active contrarians to the solution of a fixed point equation - an equilibrium.

More specifically, the situation evolves as follows: At stage $n=1$ the insurgents new actions $(x, y)$ are applied on a population apportionment $\left(A_{1}, S_{1}\right)$, meaning that the supporters and latent contrarians receive $y_{s \ell}\left(A_{1}\right) /\left(1-A_{1}\right)$ per-capita coercion and the active contrarians get $y_{a}\left(A_{1}\right) / A_{1}$ per-capita coercion. The latter results in a population reapportionment $\left(A_{2}, S_{2}\right)$, where the fraction of supporters $S_{2}$ is induced by people for whom $u\left(x, y_{s \ell}\left(A_{1}\right) /\left(1-A_{1}\right)\right) \geq \alpha$, that is,

$$
S_{2}=P\left[u\left(x, \frac{y_{s \ell}\left(A_{1}\right)}{1-A_{1}}\right) \geq \alpha\right]
$$

and the fraction of active contrarians $A_{2}$ is induced by people with $u\left(x, y_{s \ell}\left(A_{1}\right) /\left(1-A_{1}\right)\right)<\alpha$ and $u\left(x, y_{s \ell}\left(A_{1}\right) /\left(1-A_{1}\right)\right)-u\left(x, y_{a}\left(A_{1}\right) / A_{1}\right)<\beta$, that is,

$$
A_{2}=P\left[u\left(x, \frac{y_{s \ell}\left(A_{1}\right)}{1-A_{1}}\right)<\alpha ; u\left(x, \frac{y_{s \ell}\left(A_{1}\right)}{1-A_{1}}\right)-u\left(x, \frac{y_{a}\left(A_{1}\right)}{A_{1}}\right)<\beta\right] .
$$

Iterating on this process, i.e. replacing $\left(A_{i}, S_{i}\right)$ by $\left(A_{n+i}, S_{n+i}\right)$ for $i=1,2$ and for some $n=1,2, \ldots$ in the preceding argument, leads to the following result.

Proposition 1. If we assume conditions A.1, A.2, A.4, and A.5 then, for insurgents' actions $(x, y)$ and an initial population share $\left(A_{1}, S_{1}\right)$, the sequence of population apportionments $\left(A_{n}, S_{n}\right)_{n \geq 1}$ converges to a unique limit $(A, S)$ as $n \rightarrow \infty$, possibly depending on $\left(A_{1}, S_{1}\right)$, that is a solution of the root equation

$$
A=P\left[u\left(x, \frac{y_{s \ell}(A)}{1-A}\right)<\alpha ; u\left(x, \frac{y_{s \ell}(A)}{1-A}\right)-u\left(x, \frac{y_{a}(A)}{A}\right)<\beta\right],
$$

where

$$
S=P\left[u\left(x, \frac{y_{s \ell}(A)}{1-A}\right) \geq \alpha\right] .
$$

Proof. Suppose that $0 \leq A_{1} \leq A_{2}$. If $A_{1}=A_{2}$ then (5) shows that (6) holds for $A=A_{1}$. Otherwise, if $0 \leq A_{1}<A_{2}$, assumptions A.1 - A.2 mean that $y_{a}\left(A_{1}\right) / A_{1} \geq y_{a}\left(A_{2}\right) / A_{2}$ and $y_{s \ell}\left(A_{1}\right) /\left(1-A_{1}\right) \leq y_{s \ell}\left(A_{2}\right) /\left(1-A_{2}\right)$ so that

$$
u\left(x, \frac{y_{s \ell}\left(A_{1}\right)}{1-A_{1}} ; \omega\right) \geq u\left(x, \frac{y_{s \ell}\left(A_{2}\right)}{1-A_{2}} ; \omega\right)
$$

and

$$
u\left(x, \frac{y_{s \ell}\left(A_{1}\right)}{1-A_{1}} ; \omega\right)-u\left(x, \frac{y_{a}\left(A_{1}\right)}{A_{1}} ; \omega\right) \geq u\left(x, \frac{y_{s \ell}\left(A_{2}\right)}{1-A_{2}} ; \omega\right)-u\left(x, \frac{y_{a}\left(A_{2}\right)}{A_{2}} ; \omega\right)
$$

since the utility of an individual is non-increasing as the received coercion increases. Hence,

$$
I\left(u\left(x, \frac{y_{s \ell}\left(A_{1}\right)}{1-A_{1}} ; \omega\right)<\alpha(\omega)\right) \leq I\left(u\left(x, \frac{y_{s \ell}\left(A_{2}\right)}{1-A_{2}} ; \omega\right)<\alpha(\omega)\right),
$$


for each individual $\omega$, where $I(\cdot)$ evaluates to 1 when its argument is true and to 0 otherwise. Thus any individual who was a contrarian when the fraction of actives was $A_{1}$, will remain a contrarian when the fraction of actives increases to $A_{2}$. This and (8) result in

$$
\begin{aligned}
& I\left(u\left(x, \frac{y_{s \ell}\left(A_{1}\right)}{1-A_{1}} ; \omega\right)<\alpha(\omega) ; u\left(x, \frac{y_{s \ell}\left(A_{1}\right)}{1-A_{1}} ; \omega\right)-u\left(x, \frac{y_{a}\left(A_{1}\right)}{A_{1}} ; \omega\right)<\beta\right) \\
& \leq I\left(u\left(x, \frac{y_{s \ell}\left(A_{2}\right)}{1-A_{2}} ; \omega\right)<\alpha(\omega) ; u\left(x, \frac{y_{s \ell}\left(A_{2}\right)}{1-A_{2}} ; \omega\right)-u\left(x, \frac{y_{a}\left(A_{2}\right)}{A_{2}} ; \omega\right)<\beta\right)
\end{aligned}
$$

for each individual $\omega$. This implies individuals who were active when the fraction of actives was $A_{1}$ will remain active when the fraction increases to $A_{2}$. Taking expectations over the population distribution results in $A_{2} \leq A_{3}$. A standard induction argument, replacing $A_{1}$ and $A_{2}$ by $A_{n+1}$ and $A_{n+2}$, completes the proof that $\left(A_{n}\right)_{n \geq 1}$ is monotonic when $A_{1} \leq A_{2}$. The case when $A_{1} \geq A_{2}$ Fs analogously.

Since $\left(A_{n}\right)_{n \geq 1}$ is bounded and monotone, we have that the sequence $A_{n}$ converges to a unique limit, $A$, possibly depending on the initial value $A_{1}$. The expression

$$
P\left[u\left(x, \frac{y_{s \ell}(A)}{1-A}\right)<\alpha ; u\left(x, \frac{y_{s \ell}(A)}{1-A}\right)-u\left(x, \frac{y_{a}(A)}{A}\right)<\beta\right]
$$

is a continuous function of $A$. This follows from utilizing assumptions A.4 and A.5 in conjunction with Theorem 7.43 in Shapiro et al. (2009). In this case

$$
\begin{aligned}
& P\left[u\left(x, \frac{y_{s \ell}\left(A_{n}\right)}{1-A_{n}}\right)<\alpha ; u\left(x, \frac{y_{s \ell}\left(A_{n}\right)}{1-A_{n}}\right)-u\left(x, \frac{y_{a}\left(A_{n}\right)}{A_{n}}\right)<\beta\right] \\
& \quad \rightarrow P\left[u\left(x, \frac{y_{s \ell}(A)}{1-A}\right)<\alpha ; u\left(x, \frac{y_{s \ell}(A)}{1-A}\right)-u\left(x, \frac{y_{a}(A)}{A}\right)<\beta\right]
\end{aligned}
$$

as $A_{n} \rightarrow A$. Therefore,

$$
\begin{aligned}
0 \leq & \left|A_{n+1}-P\left[u\left(x, \frac{y_{s \ell}(A)}{1-A}\right)<\alpha ; u\left(x, \frac{y_{s \ell}(A)}{1-A}\right)-u\left(x, \frac{y_{a}(A)}{A}\right)<\beta\right]\right| \\
= & \mid P\left[u\left(x, \frac{y_{s \ell}\left(A_{n}\right)}{1-A_{n}}\right)<\alpha ; u\left(x, \frac{y_{s \ell}\left(A_{n}\right)}{1-A_{n}}\right)-u\left(x, \frac{y_{a}\left(A_{n}\right)}{A_{n}}\right)<\beta\right]- \\
& P\left[u\left(x, \frac{y_{s \ell}(A)}{1-A}\right)<\alpha ; u\left(x, \frac{y_{s \ell}(A)}{1-A}\right)-u\left(x, \frac{y_{a}(A)}{A}\right)<\beta\right] \mid,
\end{aligned}
$$

and (6) follows after applying (10).

As in (9), the expression $P\left[u\left(x, y_{s \ell}(A) /(1-A)\right) \geq \alpha\right]$ is continuous in $A$, so that $(7)$ follows from (4) by sending $A_{n} \rightarrow \infty$.

From a different perspective, Proposition 1 states that cycles, where the popular behavior oscillates in some range of values, are not possible. 


\subsection{Insurgent Objectives and Constraints}

Whereas in section 3.1 we assumed that $x$ and $y$ were fixed, in this section and in section 4 we examine what actions the insurgents will choose to achieve their objectives. Because of limited resources and strength, the insurgents may have constraints on the combinations of actions $x$ and $y$ they can choose. Both benefits and coercion require resources and thus the insurgents face a tradeoff between offering net-benefits and carrying out coercion. We call the set of feasible actions $x$ and $y$ the feasible action region. When defining this region, we do not need to distinguish between the coercion applied unintentionally on supporters and latents, $y_{s l}$, and the coercion applied correctly to active contrarians, $y_{a}$; the amount of resources required to coerce an individual is independent of the identity of the recipient of the coercion. We denote $x_{\max }$ as the maximum amount of net-benefits the insurgents can provide in the feasible action region (i.e., when they do not coerce, $y=0$ ). The parameter $x_{\text {max }}$ represents the upper limit on what the insurgents can give to the population. When the net-benefits are negative the impositions are greater than the benefits and the insurgents can extract resources from the population (e.g., via taxes) and increase their coercive abilities. However, there is a limit to how much the insurgents can impose on the population (e.g., based on the number of insurgents, or a minimal level of support required for the insurgency to sustain itself), and we define $x_{\text {min }}$ to be the lower bound on the net-benefits. We assume $x_{\text {min }}>-\infty$. Because the insurgents allocate their scarce resources between net-benefits and coercion, for every net-benefit $x, x_{\min } \leq x \leq x_{\max }$, there is a maximum level of coercion $y_{\max }(x)$ the insurgents can inflict. The function $y_{\max }(x)$ is non-increasing and by construction $y_{\max }\left(x_{\max }\right)=0$. See Figure 1 . We call the curve defined by the points $\left(x, y_{\max }(x)\right)$ the efficient frontier because the insurgents will choose their actions $(x, y)$ such that they fall on this curve (see Proposition 3 in section 4).

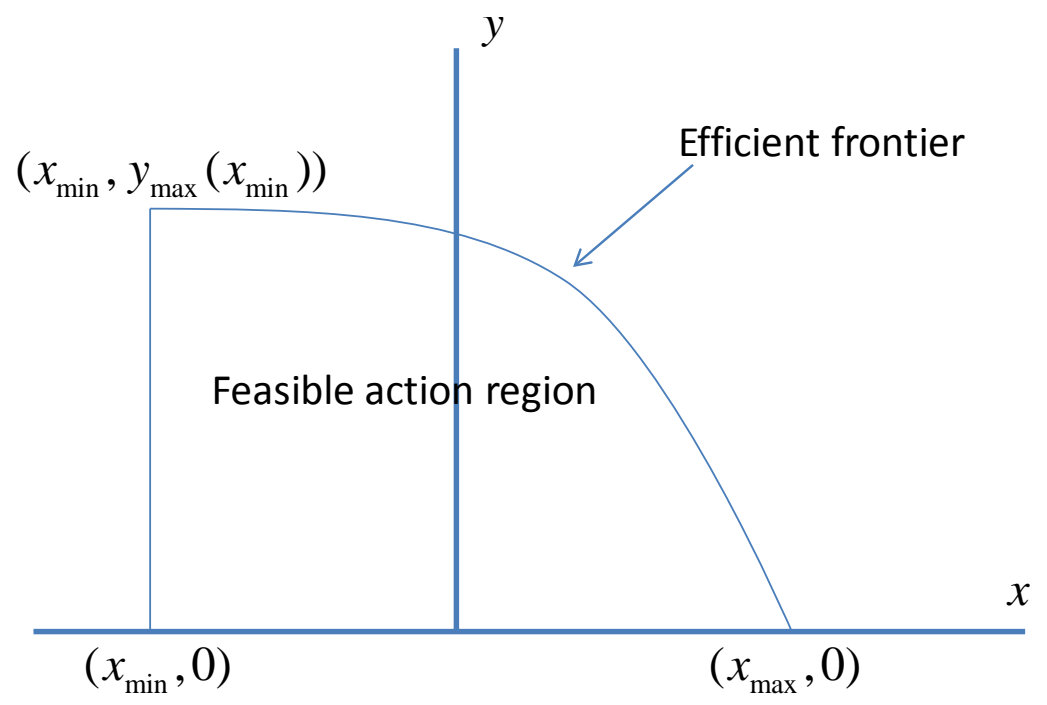

Figure 1: The feasible action region

We assume that the objectives of the insurgents are preemptive; minimize $A(x, y)$ and then maximize $S(x, y)$. This preemption assumption is reasonable because the damage that 
can be caused to the insurgents by the actions of active contrarians (e.g., providing information to the government) is much larger than the direct benefits the insurgents can gain from overt popular support. Furthermore, inspection of equation (7) reveals that if the insurgents' objective is to maximize $S(x, y)$, they would simply maximize benefits and forgo coercion (i.e., set $x=x_{\max }$ ). However, to minimize the fraction of active contrarians the insurgents would not necessarily maximize coercion $\left(x=x_{\min }, y=y_{\max }\right)$. Coercion can be a doubleedged sword; increasing coercion may convince some current active contrarians to become latent, but it also may alienate current supporters who may become active contrarians.

\section{Situational Awareness}

The behavior of the population at equilibrium depends on the situational awareness (SA) of the insurgents, as manifested in the value of the unintended coercion $y_{s \ell}(A)$. In the presence of perfect SA $y_{s \ell}(A)=0$, while no SA ("shooting in the dark") implies $y_{s \ell}(A)=(1-A) y$. The following proposition states that better SA results in fewer active contrarians.

Proposition 2. Suppose $y_{s \ell}^{(1)}(A) \geq y_{s \ell}^{(2)}(A)$ for all $A \in[0,1]$ and that assumptions A.1, A.2, and A.6 hold. Furthermore, define $A^{(1)}(x, y)$ as the limit of the sequence described in section 3.1 with initial value $A_{1}^{(1)}$ and situational awareness given by $y_{\text {sl }}^{(1)}(A)$. Define $A^{(2)}(x, y)$ and $A_{1}^{(2)}$ analogously. If $A_{1}^{(1)} \geq A_{1}^{(2)}$, then $A^{(1)}(x, y) \geq A^{(2)}(x, y)$ and $\min _{x_{\min } \leq x \leq x_{\max }} A^{(1)}(x, y) \geq$ $\min _{x_{\min } \leq x \leq x_{\max }} A^{(2)}(x, y)$ for all feasible $y$.

Proof. Suppose $A_{1}^{(2)}>0$. For an arbitrary individual $\omega$, our assumptions imply

$$
\frac{y_{s \ell}^{(1)}\left(A_{1}^{(1)}\right)}{1-A_{1}^{(1)}} \geq \frac{y_{s \ell}^{(2)}\left(A_{1}^{(1)}\right)}{1-A_{1}^{(1)}} \stackrel{A .2}{\geq} \frac{y_{s \ell}^{(2)}\left(A_{1}^{(2)}\right)}{1-A_{1}^{(2)}}
$$

so that

$$
u\left(x, \frac{y_{s \ell}^{(1)}\left(A_{1}^{(1)}\right)}{1-A_{1}^{(1)}} ; \omega\right) \leq u\left(x, \frac{y_{s \ell}^{(2)}\left(A_{1}^{(2)}\right)}{1-A_{1}^{(2)}} ; \omega\right) .
$$

Likewise, since $y_{a}^{(1)}\left(A_{1}^{(1)}\right) \leq y_{a}^{(2)}\left(A_{1}^{(1)}\right)$,

$$
\frac{y_{a}^{(1)}\left(A_{1}^{(1)}\right)}{A_{1}^{(1)}} \leq \frac{y_{a}^{(2)}\left(A_{1}^{(1)}\right)}{A_{1}^{(1)}} \stackrel{A .1}{\leq} \frac{y_{a}^{(2)}\left(A_{1}^{(2)}\right)}{A_{1}^{(2)}}
$$

so that,

$u\left(x, \frac{y_{s \ell}^{(1)}\left(A_{1}^{(1)}\right)}{1-A_{1}^{(1)}} ; \omega\right)-u\left(x, \frac{y_{a}^{(1)}\left(A_{1}^{(1)}\right)}{A_{1}^{(1)}} ; \omega\right) \stackrel{(11),(12)}{\leq} u\left(x, \frac{y_{s \ell}^{(2)}\left(A_{1}^{(2)}\right)}{1-A_{1}^{(2)}} ; \omega\right)-u\left(x, \frac{y_{a}^{(2)}\left(A_{1}^{(2)}\right)}{A_{1}^{(2)}} ; \omega\right)$.

Therefore,

$$
\begin{gathered}
P\left[u\left(x, \frac{y_{s \ell}^{(1)}\left(A_{1}^{(1)}\right)}{1-A_{1}^{(1)}}\right)<\alpha ; u\left(x, \frac{y_{s \ell}^{(1)}\left(A_{1}^{(1)}\right)}{1-A_{1}^{(1)}}\right)-u\left(x, \frac{y_{a}^{(1)}\left(A_{1}^{(1)}\right)}{A_{1}^{(1)}}\right)<\beta\right] \\
\geq P\left[u\left(x, \frac{y_{s \ell}^{(2)}\left(A_{1}^{(2)}\right)}{1-A_{1}^{(2)}}\right)<\alpha ; u\left(x, \frac{y_{s \ell}^{(2)}\left(A_{1}^{(2)}\right)}{1-A_{1}^{(2)}}\right)-u\left(x, \frac{y_{a}^{(2)}\left(A_{1}^{(2)}\right)}{A_{1}^{(2)}}\right)<\beta\right],
\end{gathered}
$$


meaning that $A_{2}^{(1)} \geq A_{2}^{(2)}$ and, by an induction argument, that $A_{n}^{(1)} \geq A_{n}^{(2)}$ for $n=1,2, \ldots$. Applying Proposition 1 now yields $A^{(1)} \geq A^{(2)}$.

For the last claim, suppose $\left(x^{*}, y^{*}\right)$ is the minimizer of $A^{(1)}(x, y)$ over the feasible set. Then $A^{(1)}\left(x^{*}, y^{*}\right) \geq A^{(2)}\left(x^{*}, y^{*}\right) \geq \min _{x \in\left[x_{\min }, x_{\max }\right]} A^{(2)}(x, y)$.

If $A_{1}^{(2)}=0$, we can consider two positive sequences, $\left(\epsilon_{m}^{(i)}\right)_{m \geq 1}$ for $i=1,2$, such that $\epsilon_{m}^{(1)} \geq \epsilon_{m}^{(2)}$ and $\epsilon_{m}^{(i)} \rightarrow A_{1}^{(i)}$ as $m \rightarrow \infty$. Clearly, Eq. (13) holds with $\epsilon_{m}^{(i)}$ in lieu of $A_{1}^{(i)}$. Sending $m \rightarrow \infty$, assumption A.6 shows that $A_{2}^{(1)} \geq A_{2}^{(2)}$.

Proposition 2 illustrates the importance of intelligence and information operations in counterinsurgency campaigns. The government needs intelligence from active contrarians to effectively engage with the insurgents. To increase the supply of these intelligence sources the government should take measures to reduce the insurgents' intelligence capabilities; that is decrease the insurgents' situational awareness. The next proposition characterizes the optimal mix of actions of the insurgents.

Proposition 3. Under assumptions A.1, A.2, A.3, A.6, and A.7, the optimal insurgent allocation lies on the efficient frontier.

Proof. For the technical steps of this proof we do not restrict $x$ and $y$ to the feasible region. We first define $x^{(1)} \leq x^{(2)}$. We next show that for any fixed $y$ that $A\left(x^{(1)}, y\right) \geq A\left(x^{(2)}, y\right)$ if the initial fraction of active contrarians satisfies $A_{1}^{(1)} \geq A_{1}^{(2)}$. Thus, when we do restrict the insurgents to the feasible region, it holds that for any interior point $(x, y)$ the insurgents are always better off increasing the benefits to the efficient frontier because the fraction of active contrarians will (weakly) decrease.

Let us initially assume that $A_{1}^{(2)}>0$. The assumptions yield,

$$
u\left(x^{(1)}, \frac{y_{s \ell}\left(A_{1}^{(1)}\right)}{1-A_{1}^{(1)}} ; \omega\right) \stackrel{A .2}{\leq} u\left(x^{(1)}, \frac{y_{s \ell}\left(A_{1}^{(2)}\right)}{1-A_{1}^{(2)}} ; \omega\right)
$$

and

$$
u\left(x^{(1)}, \frac{y_{a}\left(A_{1}^{(1)}\right)}{A_{1}^{(1)}} ; \omega\right) \stackrel{A .1}{\geq} u\left(x^{(1)}, \frac{y_{a}\left(A_{1}^{(2)}\right)}{A_{1}^{(2)}} ; \omega\right)
$$


Therefore,

$$
\begin{aligned}
& u\left(x^{(2)}, \frac{y_{s \ell}\left(A_{1}^{(2)}\right)}{1-A_{1}^{(2)}} ; \omega\right)-u\left(x^{(2)}, \frac{y_{a}\left(A_{1}^{(2)}\right)}{A_{1}^{(2)}} ; \omega\right) \\
& =\int_{-\infty}^{x^{(2)}}\left[\frac{\partial u\left(x, y_{s \ell}\left(A_{1}^{(2)}\right) /\left(1-A_{1}^{(2)}\right) ; \omega\right)}{\partial x}-\frac{\partial u\left(x, y_{a}\left(A_{1}^{(2)}\right) / A_{1}^{(2)} ; \omega\right)}{\partial x}\right] d x \\
& =u\left(x^{(1)}, \frac{y_{s \ell}\left(A_{1}^{(2)}\right)}{1-A_{1}^{(2)}} ; \omega\right)-u\left(x^{(1)}, \frac{y_{a}\left(A_{1}^{(2)}\right.}{A_{1}^{(2)}} ; \omega\right) \\
& +\int_{x^{(1)}}^{x^{(2)}}\left[\frac{\partial u\left(x, y_{s \ell}\left(A_{1}^{(2)}\right) /\left(1-A_{1}^{(2)}\right) ; \omega\right)}{\partial x}-\frac{\partial u\left(x, y_{a}\left(A_{1}^{(2)}\right) / A_{1}^{(2)} ; \omega\right)}{\partial x}\right] d x \\
& \stackrel{(14),(15)}{\geq} u\left(x^{(1)}, \frac{y_{s \ell}\left(A_{1}^{(1)}\right)}{1-A_{1}^{(1)}} ; \omega\right)-u\left(x^{(1)}, \frac{y_{a}\left(A_{1}^{(1)}\right)}{A_{1}^{(1)}} ; \omega\right) \\
& +\int_{x^{(1)}}^{x^{(2)}}\left[\frac{\partial u\left(x, y_{s \ell}\left(A_{1}^{(2)}\right) /\left(1-A_{1}^{(2)}\right) ; \omega\right)}{\partial x}-\frac{\partial u\left(x, y_{a}\left(A_{1}^{(2)}\right) / A_{1}^{(2)} ; \omega\right)}{\partial x}\right] d x \\
& \stackrel{(A .3),(A .7)}{\geq} u\left(x^{(1)}, \frac{y_{s \ell}\left(A_{1}^{(1)}\right)}{1-A_{1}^{(1)}} ; \omega\right)-u\left(x^{(1)}, \frac{y_{a}\left(A_{1}^{(1)}\right)}{A_{1}^{(1)}} ; \omega\right) .
\end{aligned}
$$

The last inequality follows because assumption A.3 implies that active contrarians receive higher per-capita coercion than latents or supporters (see condition (3)). This, in conjunction with assumption A.7, implies the expression inside the integral in the penultimate line is nonnegative and then the final inequality follows. The final inequality and Eq. (14) show that

$$
\begin{array}{r}
P\left[u\left(x^{(1)}, \frac{y_{s \ell}\left(A_{1}^{(1)}\right)}{1-A_{1}^{(1)}}\right)<\alpha ; u\left(x^{(1)}, \frac{y_{s \ell}\left(A_{1}^{(1)}\right)}{1-A_{1}^{(1)}}\right)-u\left(x^{(1)}, \frac{y_{a}\left(A_{1}^{(1)}\right)}{A_{1}^{(1)}}\right)<\beta\right] \\
\geq P\left[u\left(x^{(2)}, \frac{y_{s \ell}\left(A_{1}^{(2)}\right)}{1-A_{1}^{(2)}}\right)<\alpha ; u\left(x^{(2)}, \frac{y_{s \ell}\left(A_{1}^{(2)}\right)}{1-A_{1}^{(2)}}\right)-u\left(x^{(2)}, \frac{y_{a}\left(A_{1}^{(2)}\right)}{A_{1}^{(2)}}\right)<\beta\right],
\end{array}
$$

so that $A_{2}^{(1)} \geq A_{2}^{(2)}$. For any $n$, similar analysis yields that $A_{n}^{(1)} \geq A_{n}^{(2)}$ results in $A_{n+1}^{(1)} \geq A_{n+1}^{(2)}$. Proposition 1 then shows that $A\left(x^{(1)}, y\right) \geq A\left(x^{(2)}, y\right)$, as desired. Therefore the insurgents will maximize net-benefits for a fixed coercion level $y$.

When $A_{1}^{(2)}=0$, the proof follows the lines of the last part of Proposition 2 and is thus omitted.

The family of additive and separable utility functions, which are a general and natural class of utility functions to assign to the population in our context, satisfy the condition in A.7. In this case $u(x, y, \omega)=u_{1}(x, \omega)-u_{2}(y, \omega)$, where $u_{1}$ and $u_{2}$ are non-decreasing functions. Therefore, according to Proposition 3, the insurgents' optimal allocation must lie on the efficient frontier. 


\subsection{No Situational Awareness}

The insurgents have no situational awareness when they pick people in the population at random to coerce, so that $y_{s \ell}(A)=y(1-A)$ and $y_{a}(A)=y A$. This is the worst-case for the insurgents; the coercion is uniformly applied to the entire population and supporters, latent contrarians, and active contrarians all receive the same net-benefits and coercion from the insurgents. Thus, for any set of insurgents' actions $(x, y)$ and (random) utility function $u(x, y)$, equations (6) and (7) become

$$
\begin{aligned}
& S=P[u(x, y) \geq \alpha] \\
& A=P[u(x, y)<\alpha ; 0<\beta]
\end{aligned}
$$

The values of $S$ and $A$ defined in (16)-(17) are independent of the initial values $S_{1}$ and $A_{1}$ (see section 3.1). Since a contrarian has the same utility regardless if he is latent or active, in principle, there should be no latent contrarians because such contrarians have nothing to gain by being latent. That is, $A=C=1-S$ and $L=0$. However, it is possible that $L>0$ if some contrarians are "hard core" latents $(\beta=0)$ who would never actively aid the government even if they have nothing to lose by doing it.

In this worst-case, coercion is not a deterrence, but only serves to alienate supporters and increase the fraction of the population who are active contrarians. Because $u(x, y)$ is nondecreasing in $x$ and non-increasing in $y$, inspection of equation (17) reveals that $A(x, y)$ is non-increasing in $x$ and non-decreasing in $y$. Therefore, the optimal allocation of insurgents' resources in this case is to maximize net-benefits and impose no coercion: $(x, y)=\left(x_{\max }, 0\right)$. Obviously, rational insurgents will not apply coercion if they have no clue who is who in the population and are aware of the potential consequences of their unintended coercion.

If the population has sufficiently heterogeneous attitudes, then the function $S(x, y)$ defined by equation (16) will be continuous. More formally, utilizing assumption A.4 in conjunction with Theorem 7.43 in Shapiro et al. (2009) guarentees continuity of $S(x, y)$. Because the population can only switch between active contrarians and supporters or latent contrarians and supporters (i.e., it is not possible for an individual to switch from an active contrarian to a latent contrarian), if $S(x, y)$ is continuous in $(x, y)$ so must also be $A(x, y)$ and $L(x, y)$.

In this situation many in the population will support the insurgents. However, all contrarians will be active and will provide the government with information. The government should take actions to limit the number of supporters, knowing that the contrarians will likely be valuable intelligence sources. One option for the government is to increase the amount of benefits they provide to the population (i.e., shifting the distribution of $\alpha$ to the right), and the second option is to restrict the actions of the insurgents to limit the benefits they can provide to the population (i.e., reduce $x_{\max }$ ). An example of the second option would be to reduce or eliminate any flow of external funds and supplies to the insurgents.

\subsection{Perfect Situational Awareness}

When the insurgents have perfect SA they can accurately target their coercion on active contrarians so that the supporters and latent contrarians receive no coercion, that is, $y_{s \ell}(A)=$ 
0 and $y_{A}(A)=y$. In this case the population breakdown is determined by the equations

$$
\begin{aligned}
& S=P[u(x, 0) \geq \alpha] \\
& A=P\left[u(x, 0)<\alpha ; u(x, 0)-u\left(x, \frac{y}{A}\right)<\beta\right] .
\end{aligned}
$$

These equations represent the best-case scenario for the insurgents because the coercion is most effective. The fraction of supporters in equation (18) does not depend upon the coercion level $y$, and thus $S$ and $C$ are immediately determined only by the net-benefit level $x$. Coercion only affects the split of contrarians between active and latent.

Since the utility function $u(x, y)$ is non-increasing in $y$ (more coercion cannot increase utility) it follows from (19) that $A(x, y)$ is non-increasing in $y$. Thus, the insurgents will always choose $(x, y)$ on the efficient frontier; that is, for a fixed level of net-benefits $x$, the insurgents will always choose $y_{\max }(x)$. Notice that unlike Proposition 3 that applies to any level of SA, in the case of perfect SA we do not need A.7 to establish this property. In summary, increasing $y$ does not affect the value of $S$, and it can only decrease the fraction of active contrarians because a larger value of $y$ increases the cost of being active without increasing the cost of being latent.

To make the analysis tractable, we next assume that the nonviolent and coercive actions have an additive effect on the utility of individuals, meaning that $u(x, y)$ takes the form

$$
u(x, y)=u_{1}(x)-u_{2}(y),
$$

where $u_{1}(\cdot)$ captures the utility of benefits/impositions and $-u_{2}(\cdot)$ is the disutility produced by coercive actions. Following equations (18) and (19) the fractions of supporters and active contrarians are now given by

$$
\begin{aligned}
& S=P\left[u_{1}(x) \geq \alpha\right] \\
& A=P\left[u_{1}(x)<\alpha ; u_{2}\left(\frac{y}{A}\right)<\beta\right] .
\end{aligned}
$$

Without loss of generality we can define $u_{2}(0)=0$ in equations (21)-(22) by absorbing this term into the $\alpha$ and $\beta$ parameters. We assume that for any individual $u_{1}(\cdot)$ and $u_{2}(\cdot)$ are continuous and monotonically increasing so their inverses, $u_{1}^{-1}(\cdot)$ and $u_{2}^{-1}(\cdot)$, are well defined. Defining $\gamma=u_{1}^{-1}(\alpha)$ and $\delta=u_{2}^{-1}(\beta)$, equations (21) and (22) become

$$
\begin{aligned}
& S=P[\gamma \leq x] \\
& A=P\left[\gamma>x ; \delta>\frac{y}{A}\right]
\end{aligned}
$$

For each individual, $\gamma$ can be interpreted as a support threshold that indicates the minimum level of net-benefits $x$ that will make that individual a supporter of the insurgents. We view $\delta$ as a latency threshold, meaning that a contrarian becomes latent if the per-capita coercion is larger than $\delta$. The joint distribution of $\gamma$ and $\delta$ reflects the attitude of the population towards the actions of the insurgents and the government. This distribution may take many forms, based on social, religious and cultural factors. Individuals with small values of $\gamma$ are typically ideologically in line with the insurgents and therefore are easily pleased with relatively small 
values of net-benefits. Large values of the support threshold indicate individuals that are hard to please; the insurgents need to shower these individuals with many benefits in-order to overcome these individuals' negative attitude towards the insurgents. A small value of the latency threshold $\delta$ indicates high sensitivity to coercion while a high value of $\delta$ is associated with individuals who are not easily scared by coercion. We make the following assumption on the distribution of $\gamma$ to make the situation non-trivial: $P\left[\gamma>x_{\max }\right]>0$ and $P\left[\gamma<x_{\min }\right]>0$, meaning that there can be supporters and contrarians for all feasible values of $x$.

As stated above, the insurgents will maximize their coercive force for a fixed value of $x$ and therefore their actions $(x, y)$ will lie on the efficient frontier $\left(x, y_{\max }(x)\right)$. Thus the insurgents only choose net-benefits $x$, which determines the coercion level at $y_{\max }(x)$, and therefore $S(x)$ and $A(x)$ only depend upon $x$. Abusing notation we write $y(x)$ in lieu of $y_{\max }(x)$. As described in section 3.1, $A(x)$ is the limit of a sequence and thus depends upon the initial value of the sequence. Therefore $A(x)$ and $S(x)$ not only depend upon $x$, but also on the value of $A$ when the insurgents chose that value of $x$. This is discussed further in section 4.2.1.

\subsubsection{Basic results}

We first define the right hand side of equation (24) as a function of $x$ and $A$ :

$$
g(x, A)=P\left[\gamma>x ; \delta>\frac{y(x)}{A}\right]
$$

The fraction of active contrarians in equilibrium is obtained as a solution of the fixed point equation $A=g(x, A)$. The function $g(x, A)$ is non-decreasing in $A$ and is continuous in $A$ under assumption A.5. The nature of the solutions to the fixed point equation depends upon the joint distribution of $\gamma$ and $\delta$. Figure 2 depicts an example of $g(x, A)$ as a function of $A$.

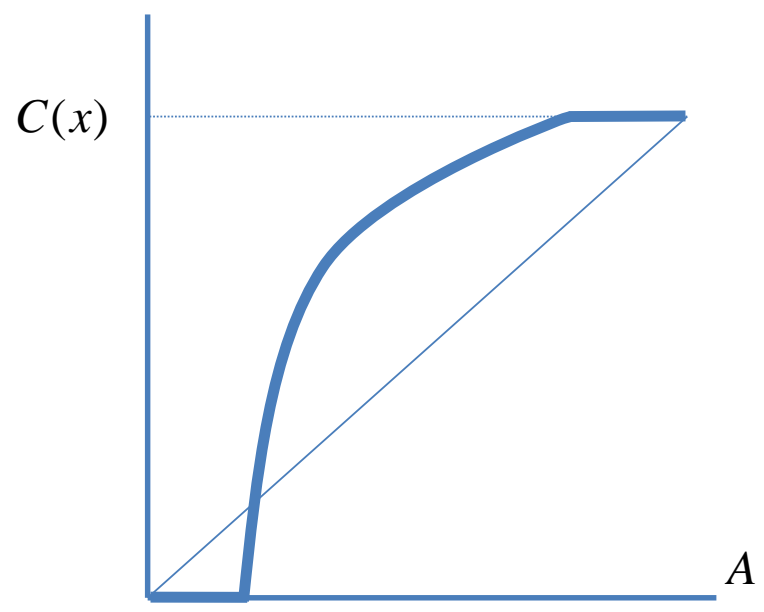

Figure 2: The $g(x, A)$ function 
When the insurgents choose a new net-benefit level $x$, the fraction of active contrarians $A$ will evolve according to the monotonic sequence $A_{n}=g\left(x, A_{n-1}\right)$, described in section 3.1, which converges to a solution of $A=g(x, A)$. As illustrated in Figure 2, there may be multiple solutions to the fixed point equation; the specific equilibrium reached in practice is determined by the initial condition $A_{1}$, which is the fraction of active contrarians at the time when the insurgents choose a new value of $x$. Each fixed point solution $A=g(x, A)$ has a basin of attraction defined by

$$
B(A)=\left\{\tilde{A} \in[0,1] \mid \lim A_{n}=A \text { where, } A_{n}=g\left(x, A_{n-1}\right), A_{1}=\tilde{A}\right\} .
$$

That is, a value $\tilde{A} \in[0,1]$ is in the basin of attraction of $A$ if the sequence $A_{n}=g\left(x, A_{n-1}\right)$ converges to $A$ when $A_{1}=\tilde{A}$. If $B(A)=A$ then the fixed point solution $A$ is a repeller; otherwise the fixed point is an attractor with a basin of attraction that may be small or large. If an equilibrium is a repeller, then a slight perturbation of the fraction of active contrarians will cause the system to evolve to a new equilibrium. Note that each $\tilde{A} \in[0,1]$ must belong to the basin of attraction of exactly one equilibrium $A$. This follows from the fact that for any $A_{1} \in[0,1]$ the sequence $A_{n}=g\left(x, A_{n-1}\right)$ monotonically approaches a unique limit, which satisfies $A=g(x, A)$ (see section 3.1).

Next we examine the existence of specific equilibrium values $A=g(x, A)$. We write $A(x)$ to denote the dependence of an equilibrium point on the net-benefits level $x$. If the insurgents maximize the net-benefits, $x=x_{\max }$, and execute no coercion, then equation (24) simplifies to $A(x)=C(x)$ and the only equilibrium is for all contrarians to be active. This result is straightforward (no coercion implies no latent contrarians) and independent of the situational awareness of the insurgents. Below we present a sufficient condition for the existence of the best equilibrium for the insurgents: $A(x)=0$.

Proposition 4. If there exists an $x_{0}$ such that $P\left[\gamma>x_{0} ; \delta>M\right]=0$ for some finite $M$, then $A(x)=0$ is an attractor equilibrium for all $x_{0} \leq x<x_{\max }$

Proof. If $P\left[\gamma>x_{0} ; \delta>M\right]=0$, then inspection of equation (25) yields

$$
g\left(x_{0}, A\right)=0 \quad \text { for } 0 \leq A<\frac{y\left(x_{0}\right)}{M}
$$

Therefore $\left[0, \frac{y\left(x_{0}\right)}{M}\right]$ is a subset of $B(0)$. That is the basin of attraction for the equilibrium $A\left(x_{0}\right)=0$ has positive mass as long as $y\left(x_{0}\right)>0$ (which by assumption holds for any $\left.x_{0}<x_{\max }\right)$. Thus $A\left(x_{0}\right)=0$ is an attractor equilibrium. The same argument applies for any $x_{0} \leq x<x_{\max }$ because $y(x)>0$ and $P[\gamma>x ; \delta>M]=0$. The latter condition holds because $P[\gamma>x ; \delta>M] \leq P\left[\gamma>x_{0} ; \delta>M\right]$.

Proposition 4 states that if the contrarians in the population have bounded latency thresholds then $A(x)=0$ is an attractor equilibrium. If, in addition to the assumptions made in Proposition 4, the upper bound $M$ is such that $\frac{y\left(x_{0}\right)}{C\left(x_{0}\right)}>M$ then $A\left(x_{0}\right)=0$ is the only equilibrium because the per-capita coercion would be more than any contrarian could bear. In this case the insurgents would want to choose $x_{0}$ to stamp out all active opposition. When non-zero equlibria exist for every $x_{\min } \leq x<x_{\max }$, the insurgents may still successfully coerce all contrarians (i.e., attain the equilibrium $A(x)=0$ ). If this occurs, 
then Proposition 4 guarantees the system will remain at $A(x)=0$ for any increase in the net-benefit level below $x_{\max }$. Therefore the insurgents should then maximize net-benefits (technically set $x=x_{\max }-\epsilon$ ) to capture as much support as possible while remaining confident that no intelligence will flow to the government. This is the worst case scenario for the government. If they ever completely lose all intelligence sources, it may be difficult to regain them.

If there are individuals in the population that are very sensitive to coercion (i.e., have small latency thresholds), then it is not possible to have the equilibrium $A(x)=C(x)$. More precisely, if there exists contrarians in the population such that $\delta(\omega)<y(x) / C(x)$, then $g(x, C(x))<C(x)$. In this case even if all contrarians are active, the per-capita coercion each would receive would be greater than the latency thresholds of some contrarians. If however all the contrarians are willing to bear this coercion (i.e., for all contrarians $\delta(\omega)>$ $y(x) / C(x))$ then $g(x, C(x))=C(x)$ and the best equilibrium for the government would exist: $A(x)=C(x)$. Whether the system evolves to one of the extreme equilibriums $A(x)=0$ or $A(x)=C(x)$ (or a mixed one in between) depends upon the initial value of $A$.

\subsubsection{Tipping Points}

Tipping points are a specific type of discontinuity in the function $A(x)$. A small change in $x$ by the insurgents may cause the equilibrium fraction of active contrarians to shift significantly. Tipping points can be beneficial or detrimental to the government; that is $A(x)$ may jump up or drop down abruptly as a result of a small change in the net-benefits (and the resulting coercion - see Proposition 3). Mathematically, tipping points are bifurcations that occur when, for a certain equilibrium value $A(x)$, we have $\frac{d}{d A} g(x, A(x))=1$ (Hirsch et al., 2003). The actual level of net-benefits $x$ where tipping points occur depends upon the functional form of the efficient frontier $y(x)$ and the nature of the bivariate distribution of $(\gamma, \delta)$. There may be multiple tipping points in $x_{\min } \leq x \leq x_{\max }$ or there may be none.

Tipping points may arise in several situations. As an example, consider the case where: (i) $g(x, A)$ is continuous and concave in $A$ for all feasible net-benefits $x$; (ii) there are feasible net-benefits $x$ with $g(x, \cdot)=0$ in a neighborhood of $A=0$; and (iii), for each feasible $x$ there exists a value of $A$ such that $\partial g(x, A) / \partial A=1$. From the definition of $g$, condition (i) happens when the contrarian population is overly insensitive to coercion for any level of net benefits $x$, and condition (ii) is satisfied when the latency thresholds of the contrarian population become bounded as the net benefits increase (see Proposition 4). In this case, increasing the net-benefits causes the active contrarians who are most insensitive to coercion to switch to supporters, while the remaining active contrarians bear increasingly more percapita coercion.

This scenario is illustrated in Figure 3. The fraction of active contrarians at equilibrium satisfies $A=g(x, A)$, which occurs at the intersection of the 45 degree line and the concave curve in figure $3(\mathrm{a})$. If the insurgents change their actions such that the concave curve moves southeast and detaches from the 45 degree line (as in Figure 3(b)), then a domino effect will occur leading to the elimination of all active contrarians. After this change in actions, the fraction of active contrarians will evolve to a new equilibrium according to the dynamics described in section 3.1. Initially the cost of being an active contrarian increases, and therefore some of the active contrarians become latent. This appears in Figure 3(b) 
where the fraction of actives drops from $A_{1}=A^{*}$ to $A_{2}$. This decrease in the number of active contrarians means that the remaining active contrarians face more per-capita coercion. Some of the remaining active contrarians then decide that it is too costly to remain active and they become latent (the fraction of actives drops from $A_{2}$ to $A_{3}$ in Figure $3(\mathrm{~b})$ ). A cascade effect ensues where the increased cost drives actives to become latents, which only further increases the cost of being active causing more individuals to switch from active to latent. The net result is that all active contrarians become latent, and the government no longer has intelligence sources in the population. The dashed line in Figure 3(b) illustrates the path the population of active contrarian follows as their fraction quickly drops from $A^{*}$ to 0 . If the population equilibrium is defined by Figure 3(a), the government would want to do everything it could to prevent the system from evolving to Figure 3(b) and the resulting large scale population shift.

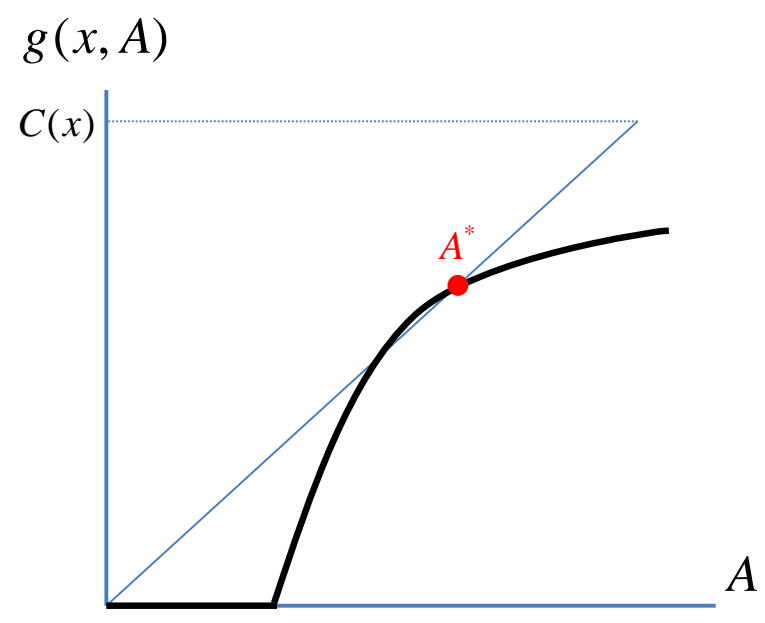

$g(x, A)$

$A$

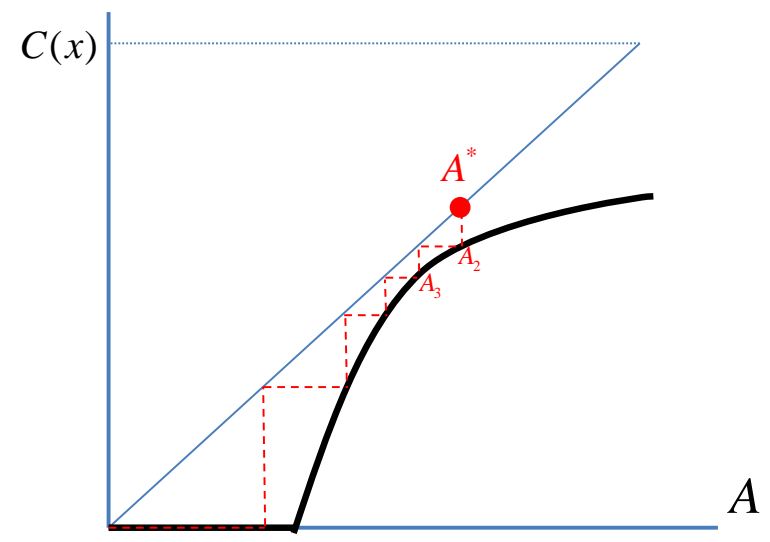

(a) In equilibrium there is a positive fraction of active contrarians $A^{*}$

(b) A change in the insurgent's actions leads to a slight increase in the cost of being active, which shifts the black curve down. This leads to a dramatic change in the fraction of active contrarians as the equilibrium value cascades down from $A^{*}$ to 0. $A_{1}=A^{*}$ and $A_{2}$ and $A_{3}$ are the vertical components of the indicated points on the solid black curve.

Figure 3: Tipping point illustration

In the scenario described by Figure 3, a tipping point that is beneficial to the government (i.e., a significant increase in the fraction of active contrarians) does not exist. However, in practice those discontinuities may exist (a figure representing such a scenario might consist of a a convex curve above the 45 degree line). 


\subsubsection{Optimal insurgent actions}

The insurgents want to choose the level of net-benefits to minimize the fraction of active contrarians. However for a given net-benefit level $x$, there may be multiple solutions to the fixed point equation $A=g(x, A)$, and the equilibrium $A(x)$ reached in practice depends upon the initial condition (see section 3.1). Therefore, the insurgents' optimal decision must account for the initial condition. It appears difficult to derive general results about the insurgents' global optimal action. However, this analysis can be done on a case by case basis for specific realizations of the efficient frontier, the bivariate distribution for $(\gamma, \delta)$, and the initial conditions of the population fractions. We instead examine how the insurgents behave optimally, in a local sense, given the system is already at some equilibrium state $A(x)$.

If assumptions A.4-A.6 hold then there exists a bivariate density $f(v, w)$ that defines the joint distribution of $(\gamma, \delta)$. We can rewrite equation (25)

$$
g(x, A)=P\left[\gamma>x ; \delta>\frac{y(x)}{A}\right]=\int_{x}^{\infty} \int_{\frac{y(x)}{A}}^{\infty} f(v, w) d w d v
$$

In this case $g(x, A)$ is continuous in $x$ and $A$ if $y(x)$ is a continuous function of $x$. The function $g(x, A)$ also is differentiable in $x$ and $A$ at all values except where there are discontinuities in the density at $f(x, y(x) / A)$ (e.g., a bivariate uniform; see figures 2 and 3 for illustrations of this non-differentiability). For a specific equilibrium $(x, A(x))$ we can analyze how this equilibrium changes locally (i.e., while remaining in the same basin of attraction) via the implicit function theorem, thus providing insights into how the insurgents should change their actions. When the insurgents increase net-benefits $x$, there are two effects that change the equilibrium fraction of active contrarians $A(x)$. The increase in net-benefits causes some active contrarians to switch to supporters. However, the increase in net-benefits results in a reduction in coercion, which initially reduces the cost of being active, and thus some latent contrarians may become active. $A(x)$ increases or decreases with $x$ depending on which of these two effects dominates. The derivative of $A(x)$ is given by (see Chapter 7 of Marsden and Hoffman (1993))

$$
\frac{d A}{d x}=\left.\frac{\partial g(x, A) / \partial x}{1-\partial g(x, A) / \partial A}\right|_{(x, A(x))} .
$$

We only consider attractor equilibria because those are the type that are found in practice. Attractor equilibria have the property that $|\partial g(x, A) / \partial A|<1$ (Hirsch et al., 2003). Recall that $g(x, A)$ is non-decreasing in $A$ (see equation (25)) and thus we have the condition $\partial g(x, A) / \partial A<1$. Therefore to analyze how $A(x)$ changes with $x$ we only need to consider the numerator $\partial g(x, A) / \partial x$. Differentiating (26) yields

$$
\frac{\partial g(x, A)}{\partial x}=\underbrace{-\frac{y^{\prime}(x)}{A} \int_{x}^{\infty} f\left(v, \frac{y(x)}{A}\right) d v}_{\text {additional actives due to less coercion }}-\underbrace{\int_{\frac{y(x)}{A}}^{\infty} f(x, w) d w}_{\text {fewer actives due to additional net-benefits }} .
$$

This derivative consists of two intuitive terms that we have labeled. These two terms represent the two effects described above that determine how the fraction of active contrarians changes with an increase in net-benefits $x$. Thus the insurgents should change the level of 
net-benefits according to the following policy:

$$
\begin{array}{cc}
\text { Increase benefits if } & -\frac{y^{\prime}(x)}{A} \int_{x}^{\infty} f\left(v, \frac{y(x)}{A}\right) d v<\int_{\frac{y(x)}{A}}^{\infty} f(x, w) d w . \\
\text { Decrease benefits if } & -\frac{y^{\prime}(x)}{A} \int_{x}^{\infty} f\left(v, \frac{y(x)}{A}\right) d v>\int_{\frac{y(x)}{A}}^{\infty} f(x, w) d w .
\end{array}
$$

From Eqs. (27)-(29), we see that when $y^{\prime}(x)$ (the elasticity of coercion with respect to benefits) is nearly nil, then Eq. (28) holds, and the insurgents should increase the level of netbenefits because they can gain supporters without losing much coercive power. Conversely, if the elasticity is extremely large, $y^{\prime}(x) \ll 0$, then Eq. (29) applies and a small increase in benefits results in a significant drop in the coercive power. In this case, the insurgents should improve their situation by decreasing benefits and increasing coercion.

The marginal densities in equation (27) represent individuals who are close to their threshold values. If $\delta(\omega)$ is bounded away from $y(x) / A$ for most $\omega$ in the population, then there are few contrarians close to their latency threshold. This implies that $f(v, y(x) / A) \approx 0$ for all $v$ and thus changing coercion has little effect. Therefore, the insurgents should increase benefits because the right hand side in Eq. (28) dominates. An example of this situation is a large fraction of the population having either a very large latency threshold or a very small latency threshold, with only a small minority having a latency threshold in between. In this case there is a large range of coercion values that yield nearly the same latent/active proportion. The insurgents can easily decrease $A$ by increasing benefits to entice active contrarians to become supporters. Similar analysis yields that if there are few individuals near their support threshold (i.e., $f(x, w) \approx 0$ for all $w$ ) the insurgents should increase coercion.

When the population consists primarily of people with large support thresholds and a heterogeneous range of latency thresholds, the leftmost integral dominates the rightmost integral in Eq. (27). Hence, if $\left|y^{\prime}(x)\right| / A \geq 1$, Eq. (29) applies and the insurgents should decrease benefits and increase coercion. The insurgents should continue to increase coercion until either $\left|y^{\prime}(x)\right| / A$ becomes small or there is a tipping point and the equilibrium moves to a new basin of attraction. Thus the only way to prevent a tipping point in this case is if $y^{\prime}(x)$ approaches 0 faster than $A$, meaning that the coercive capabilities become inelastic faster than the fraction of people receiving the coercion $(A)$ decreases. In any case, the insurgents are likely to ramp up their coercive actions at the expense of their non-violent actions. Knowing this, the government can expect to find a relatively large fraction of contrarians.

On the other hand, when the population consists of people with large latency thresholds and a heterogeneous range of support thresholds, the rightmost integral dominates the leftmost integral in Eq. (27). In this case most people are relatively insensitive to coercion. If $\left|y^{\prime}(x)\right| / A \leq 1$ we fall in the scenario of Eq. (28) and the insurgents can do better by increasing benefits. However, when the insurgents' feasible set is concave, $y^{\prime}(x) / A \rightarrow-\infty$ as $x$ increases and $A$ decreases, and no tipping may occur because the situation switches to the scenario described by Eq. (29). 


\section{Conclusions and Policy Implications}

In this paper we examine how popular support changes with insurgent actions. The main advantages of the insurgents are their elusiveness (their signature is small) and the fact that typically they are grass roots organizations who evolve from the population and thrive on its support. However, the reliance of the insurgents on the civilian population is also their greatest vulnerability; they must maintain the population's support or at least ensure the reluctance of the population to cooperate with the government. Once the insurgents lose the hearts and minds of the population and the ability to stifle dissent, they are doomed because (a) they will no longer receive the support they need from the population and (b) information, the most precious asset in an insurgency situations, will start flowing to the regime. The regime will utilize the information to increase the signature of the insurgency and thus will be able to more effectively combat the insurgents. Thus, the civilian population is a key factor in shaping an insurgency situation.

Coercion plays a central role in insurgency situations, and situational awareness determines how well the insurgents target their coercion. If the insurgents have a high level of situational awareness, coercion is an effective tool to control the population. Therefore, the government should take actions to reduce the insurgents' situational awareness (e.g., by spreading mis-information) so that the number of active contrarians increases.

When the insurgents have perfect situational awareness, tipping points may occur where small changes in the actions of the insurgents can drastically change the amount of active support for the government. The government should be aware of potential tipping points that lead to the elimination of most active contrarians and attempt to avoid situations that lead to them. Even if the insurgents have perfect situational awareness, it is possible that all of the contrarians will provide intelligence to the government. For this situation to occur the government must limit the coercing power of the insurgents (e.g., by limiting the flow of illegal arms flowing to the insurgents) and influence the population to be more willing to bear coercion (e.g., by paying them off).

If the insurgents have no situational awareness then coercion has no benefit and only serves to alienate their supporters. Rational and intelligent insurgents will therefore not use coercion and maximize the benefits they provide to the population to garner as much support as possible. In this situation there may not be many contrarians, but all of them will be active and willing to provide intelligence to the government. The government should take measures to increase the number of contrarians (e.g., increase the benefits they provide by building more hospitals and infrastructures) and foster these intelligence sources.

This work builds upon other modeling efforts in several ways. Unlike the work of Akerlof and Yellen (1994) and Berman et al. (2008), our population is a heterogeneous mix of individuals. Furthermore, we account for latent contrarians who are successfully coerced, whereas other "peasant" models (e.g., Popkin (1979) and Lichbach (1994)) consider the population as binary: supporters or non-supporters. These latent contrarians may play an important role in an insurgency situation because the insurgents may overestimate their strength if they mistakenly believe that latent contrarians are supporters. Finally our most significant contribution is accounting for imperfect targeting of coercion via situational awareness. Coercion is a double-edged sword: if targeted correctly it will stifle the flow of information to the government, but if targeted poorly the insurgents may drive the population to the 
government. Modeling the situational awareness as a dynamic factor that depends upon the population's behavior can lead to feedback effects that cause dramatic shifts in popular support.

\section{References}

A. Washburn and M. Kress, Combat Modeling, Springer, Dordrecht, Netherlands, 2009.

J. Farley, Evolutionary Dynamics of the Insurgency in Iraq: A Mathematical model of the battle for hearts and minds, Studies in Conflict \& Terrorism 30 (2007), 947-962.

J. Epstein, Modeling Civil Violence: An Agent-based Computational Approach, Proceedings of the National Academy of Sciences 99 (2002), 7243-7250.

R. Bhavnani, D. Miodownik, and J. Nart, REsCape: an agent-based framework for modeling resources, ethnicity, and conflict, Journal of Artificial Societies and Social Simulation 11 (2008).

C. Cioffi-Revilla and M. Rouleau, MASON RebeLand: An Agent-Based Model of Politics, Environment, and Insurgency1, International Studies Review 12 (2010), 31-52.

M. Granovetter, Threshold Models of Collective Behavior, American Journal of Sociology 83 (1978), 1420-1443.

M. Kleiman and B. Kilmer, The Dynamics of Deterrence, Proceedings of the National Academy of Sciences 106 (2009), 14230-14235.

D. Card, A. Mas, and J. Rothstein, Tipping and the Dynamics of Segregation, Quarterly Journal of Economics 123 (2008), 177-218.

M. Kress and R. Szechtman, Why Defeating Insurgencies is Hard: The Effect of Intelligence in Counterinsurgency Operations - A Best Case Scenario, Operations Research 57 (2009), $578-585$.

E. Berman, J. Shapiro, and J. Felter, Can Hearts and Minds be Bought? The Economics of Counterinsurgency in Iraq, 2008. NBER WP \#14606 December 2008.

L. Condra, J. Felter, R. Iyengar, and J. Shapiro, The Effect of Civilian Casualties in Afghanistan and Iraq, 2010. NBER Working Paper, January 2010.

G. Akerlof and J. Yellen, Gang Behavior, Law Enforcement, and Community Values, in Values and Public Policy, H. Aaron, T. Mann, and T. Timothy, eds., Brookings, Washington, DC (1994) pp. 173-210, pp. 173-210.

T. Kuran, Sparks and Prairie Fires: A Theory of Unanticipated Political Revolution, Public Choice 61 (1989), 41-74.

F. Zagare and D. Kilgour, Perfect Deterrence, Cambridge University Press, Cambridge, 2000. 
A. Shapiro, D. Dentcheva, and A. Ruszczynski, Lectures on Stochastic Programming: Modeling and Theory, Society for Industrial and Applied Mathematics, Philadelphia, 2009.

M. Hirsch, S. Smale, and R. Devaney, Differential Equations, Dynamical Systems, and an Introduction to Chaos, Academic Press, San Diego, CA, 2003.

J. Marsden and M. Hoffman, Elementary Classical Analysis, W.H. Freeman and Company, New York, 1993.

S. Popkin, The Rational Peasant: The Political Economy of Rural Society in Vietnam, University of California Press, Berkeley, CA, 1979.

M. Lichbach, What makes Rational Peasants Revolutionary?: Dilemma, Paradox, and Irony in Peasant Collective Action, World Politics 46 (1994), 383-418. 